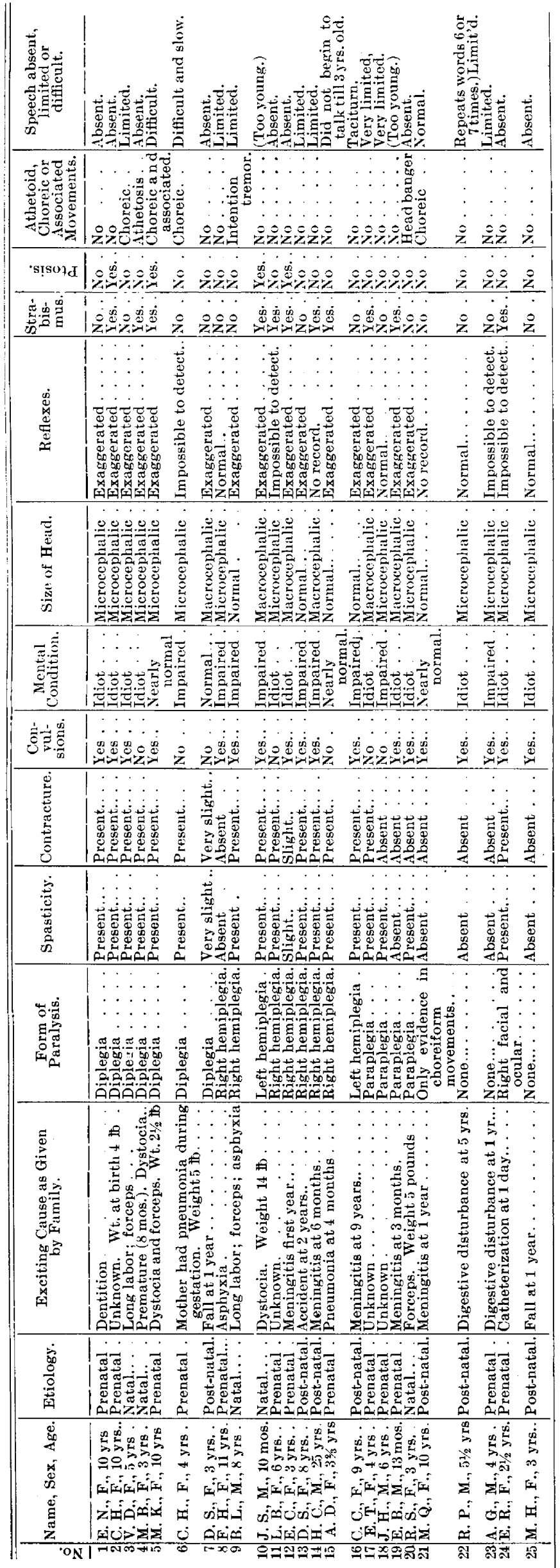

ing could be brought about in institutions than at home. There are certain exceptions to this rule, for, under proper surroundings, individual management, I think, can be carried out better at home, or in a very small institution in which only four or five children are taken. As to the circulatory disturbances, I do not think that Dr. Taylor has proved his point. It seems to me that they are more probably the result of a cerebral defect reacting through the vascular centers in the brain. Much more can be said about infantile cerebral palsy. I think it would be an excellent rule for the general practitioner to ex. amine every child brought to him suffering from epilepsy for evidence of infantile cerebral palsy. These children may make a certain amount of recovery, and the condition of partial paralysis may not be manifested sufficiently to attract the patient's attention, until symptoms of epilepsy are developed, either in the form of petit mal or grand mal. The most unsatisfactory epilepties to treat are those in whom the epilepsy is a terminal condition of the cerebral palsy.

Dr. J. P. Crozer Griffitr, Philadelphia-'The mistake is very commonly made of calling many cases chorea, Friedreich's ataxia, etc., which are really due to cerebral injuries in early life.

I wish to call attention to the rarity of tremor as a postcerebral symptom. I notice that Osler, in his book on cerebral palsies, reports, out of all his cases, only one of post-hemiplegic tremor. I have myself seen only one case. It is certainly a comparatively rare condition, whereas the spastic conditionsthe cases of single or double choreiform movements-are undoubtedly very common indeed.

I can heartily indorse what Dr. Leszynsky has said about the relation of cerebral palsy to epilepsy.

Dr. J. Madison Taylor, Philadelphia-In reply to Dr. Leszynsky's criticism of Wilmarth's assertion that the best treatment for defective children is to be had in institutions for the management of imbeciles, I desire to say that at one time I entertained the same opinion, because there was reason to be lieve, from analogy, that home treatment should be the best. I wish to say now that experience has taught me differently, and I am convinced the best results can be had by systematized and specialized instruction among children of the defective classes, with others of similar grade alongside of them as stimulants to effort. It is easy to imagine that the opportunities afforded by limitless wealth might give an equal or better training at home, but I am sure that the average case can be better handled in institutions properly equipped for such in struction. 'The subject is most important, and one on which the physician is constantly consulted, and he needs to form clear concepts on the matter.

DR. CotTon-We are all anxious to hear what those who are conducting an educational system for this unfortunate clase have to report. With one exception, these twenty-five cases were taken from my clinical records of the past year. The exception is the case represented by photograph No. 14. The child had meningitis at 6 months. There were repeated convulsions, and a semi-comatose condition supervened, which lasted four days. At 12 years of age the child was sent to the School for Feeble-Minded in Illinois. He improved there so that he could play in a band and could use certain tools. The outlook for that case was at first gloomy, but the results have been such that $\mathrm{I}$ believe the importance of educational work can not be overestimated.

\title{
THE MILK-CONTROL IN THE KAISER UND KAISERIN FRIEDRICH HOSPITAL IN BERLIN.*
}

DR. SOMMERFELD.

ASsISTANT CHEMIST AT THE HOSPITAL. BERLIN.

In this report I shall deal with the determination of temperature, the specific weight, the chemical analysis of foreign matter and the bacteriologic examination.

* Presented to the Section on Diseases of Children, at the Fifty-first Annual Meeting of the American Medical Association, held at Atlantic City, N. J., June 5-8, 1900. 
When the milk is brought into the hospital, the dairymaid takes the temperature of the milk, and makes a record of it, which is filed in the journal of the laboratory at the end of each month. The temperature shows whether the milk has been properly cooled. It should not have a higher temperature than $10 \mathrm{C}$. If it should be higher the sender is notified, who must remedy this, and if it occurs again he is subjected to a fine. The specific weight is obtained by means of an exact areometer. If there is any doubt we first take the specific gravity of full milk, allow the cream to rise in a cylinder, remove the skimmed milk by means of a faucet near the bottom of the cylinder and again take the specific gravity. After a great many examinations, we find that full milk from our stable has a specific gravity of 1029 to 1032 ; and skimmed milk of 1032 to 1038. By testing the acidity of the milk we frequently can determine its freshness in suspicious milk delivered. The acidity is determined by a sample taken from a can, a second sample being put into a thermostat and allowed to remain there for one hour at a temperature of $37 \mathrm{C}$. If there is any difference between the two, the milk is at once questioned, and regarded as not being fresh. To determine this, 25 c.c. of milk are mixed with eight to ten times as much distilled water, to which 1 c.c. of a 2 per cent. alcoholic phenolphthalein solution has been added. To this is added a solution of barium hydrate, $10.69 \mathrm{gm}$. of baryta to a liter of water. This is filtered until it leaves a faintly red color. To secure this with our infants' milk we need from 15.8 to 17.5 c.c. of barium solution, whereas with the ordinary milk we need from 16 to 19 c.c.

Exact chemical and also bacteriologic examinations are made at different times, or when specially called for. Through sudden and unexpected taking of samples we obtain valuable analytical data. Such analyses are made for the determination of dry substances, fats, albumin, sugar, mineral salts, acidity and specific gravity. Dry substances and fats are determined at least once a week. The first is accomplished by subjecting 10 c.c. to a temperature of $110 \mathrm{C}$. for twenty-four hours in a platinum vessel. Fats are determined by the Soxhlet's areometric method, or after the Libermann-Szekely-Weiss method: In a small glass-stoppered cylinder 50 c.c. of milk are placed, to which 5 c.c. of caustic potash, 50 c.c. of petroleum ether-low boiling - and 50 c.c. of alcohol (96 per cent.) are added and shaken three times, for five minutes each time. The whole layer of fat is contained in the petroleum-ether, from which all liquid parts can be removed with the pipette and evaporated to dryness. The dried residue which remains after being subjected to a temperature of $105 \mathrm{C}$. is weighed and the comparative amount estimated. Our infants' milk during the last three years has contained 3.26 per cent. of fat.

To determine the amount of milk impurities we examine several times each week. This is done after the method of Renck-Stutzer, improved by Sommerfeld. This consists of a jar with a stopper, containing about 11/2 liters, having an additional small bucket on its side which ends in a faucet. All sharp corners serve as dirtreceivers, and have been avoided, therefore the apparatus is rounded from top to bottom. This is filled with a known quantity of milk, and is kept standing for about two hours. By this time the foreign matter has settled and sedimented in the small compartments. The clear milk is now drawn off by the faucets on the sides. The attached utensil is taken off and the milk, combined with the dirt, put into a goblet, diluted with water, and then put into a filter, which has previously been weighed. This is washed over and over again with distilled water until the filtered portion is perfectly clean, then washed once with alcohol, and once with ether, and lastly dried at a temperature of $110 \mathrm{C}$. Thanks to the thorough cleanliness, the milk for nurslings seldom contains doubtful masses of dirt. As the cream rises in the apparatus while standing, we utilize the same opportunity for determining the specific gravity of the skimmed milk, since this runs off first on opening the faucet.

The bacteriologic examination of the milk is made with a view to determine the character of the bacteria present. The determination of the various forms is made in special instances, e. g., with the milk of newlyacquired cows, and when any special cow is suspected. A great many bacilli and cocci are utilized for cultures and animal experiments, to see how they affect sterile milk and to prove their harmlessness. As the cows are under the supervision of physicians, and as the milk is never used in the raw state, we have seldom occasion to examine the milk for pathogenic micro-organisms, since it is a known fact that pathologic bacteria are destroyed at $100 \mathrm{C}$. Nevertheless, we convince ourselves that none is present, especially the tubercle bacillus, by injecting guinea-pigs at monthly intervals with milk sediment procured by centrifuging. Up to the present we have found none that are pathogenic. To count the number of germs it is necessary to dilute 0.1 c.c. of milk with sterile water, the milk being taken from the cans in a sterilized pipette, and, depending on the time of the year, to inoculate either nutrient gelatin or nutrient agar therewith.

The counting is done by means of Petri dishes, which are used in making the cultures, the under side divided into squares. The gelatin plates are put into a temperature of 22 C., and are counted after twenty-four to forty-eight hours, whereas the agar plates at the sane temperature are counted after twenty-four hours. Samples of the milk are taken from the sterilizer and put into the thermostat at a temperature of $37 \mathrm{C}$., to vrove how long the milk will keep. Milk specimens which do not curdle during the first twenty-four hours shows that the lactic acid bacilli are killed. As the milk is used within ten hours after the sterilization, in the wards, of the hospital, we take samples of milk from the ice after eight hours. The negative results of our examination prove that the sterilization has been satisfactorily performed, although we frequently see, on the other hand, that the milk could hardly be classed as germless.

\section{THE MIIK-SUPPLY IN THE KAISER UND KAISERIN FRIEDRICH HOSPITAL IN BERLIN.*}

\author{
PROF. DR. A. BAGINSKY. \\ DIRFCTOR OF THE HOSPITAL. \\ BERLIN.
}

The attention given by American physicians to milksupply and milk-sterilization for nurslings prompts me to give my experience in this regard, for I believe that in our hospital we have reached the highest point attainable on this subject. Depending on our milksupply from neighboring stables, and having noticed the inadequacy of this supply, we made it a point to acquire such a stable for the hospital, especially since we, through an arrangement with the municipal authorities, were called upon to treat a large number of sick nurs-

* Presented to the Section on Diseases of Children, at the Fifty-first Annual Meeting of the American Medical Association, held at Atlantic City. N. J., June 5-8, 1900. 